\title{
Population Pharmacokinetic Model of the Pregabalin-Sildenafil Interaction in Rats: Application of Simulation to Preclinical PK-PD Study Design
}

\author{
Gregor Bender, ${ }^{1}$ James Gosset, ${ }^{2}$ Jeff Florian, ${ }^{3}$ Keith Tan, ${ }^{2}$ Mark Field, ${ }^{2}$ Scott Marshall, ${ }^{2}$ Joost DeJongh, ${ }^{4}$ \\ Robert Bies, ${ }^{3}$ and Meindert Danhof ${ }^{1,4,5}$
}

Received April 14 2009; accepted July 8 2009; published online August 11, 2009

\begin{abstract}
Purpose. Preliminary evidence has suggested a synergistic interaction between pregabalin and sildenafil for the treatment of neuropathic pain. The focus of this study was to determine the influence of sildenafil on the pharmacokinetics (PK) of pregabalin with the objective of informing the design of a quantitative pharmacodynamic (PD) study.

Methods. The pharmacokinetics were determined in rats following 2-hr intravenous infusions of pregabalin at doses of $4 \mathrm{mg} / \mathrm{kg} / \mathrm{hr}$ and $10 \mathrm{mg} / \mathrm{kg} / \mathrm{hr}$ with and without a sildenafil bolus $(2.2 \mathrm{mg})$ and steady state infusion $(12 \mathrm{mg} / \mathrm{kg} / \mathrm{hr}$ for $6 \mathrm{~h})$. This PK model was utilized in a preclinical trial simulation with the aim of selecting the optimal sampling strategy to characterize the PK-PD profile in a future study. Eight logistically feasible PK sampling strategies were simulated in NONMEM and examined through trial simulation techniques.

Results. A two-compartment population PK model best described pregabalin pharmacokinetics. Significant model covariates included either a binary effect of sildenafil administration $(30.2 \%$ decrease in clearance) or a concentration-dependent effect due to sildenafil's active metabolite.

Conclusions. Analysis of simulations indicated that three post-PD samples had the best cost/benefit ratio by providing a significant increase in the precision (and minor improvement in bias) of both PK and PD parameters compared with no PK sampling.
\end{abstract}

KEY WORDS: neuropathic pain; optimal sampling; synergistic interaction; trial simulation.

\section{INTRODUCTION}

Neuropathic pain represents an area of largely unmet medical need, with significant impact on health-related quality of life (1). At present, neuropathic pain is treated with a variety of different medications with various mechanisms of action (2-7). Current treatments for neuropathic pain achieve some relief from the unrelenting pain, leaving significant room for improvement (8). An intriguing question is whether a greater effectiveness can be obtained by using rational combinations of drugs $(9,10)$. The optimization of a rational drug combination represents a major challenge (11).

Pharmacokinetic-pharmacodynamic (PK-PD) modeling has been recognized as a promising tool for preclinical drug development including rational drug combinations $(12,13)$. In investigations on PD interactions, it is important to first account

Financial support for this project was received from Pfizer, Global Research \& Development, Sandwich, United Kingdom

\footnotetext{
${ }^{1}$ Leiden-Amsterdam Center for Drug Research, Division of Pharmacology, Leiden University, Leiden, The Netherlands.

${ }^{2}$ Pfizer, Global Research \& Development, Sandwich, United Kingdom.

${ }^{3}$ University of Pittsburgh, Pennsylvania, USA.

${ }^{4}$ LAP\&P Consultants BV, Leiden, The Netherlands.

${ }^{5}$ To whom correspondence should be addressed. (e-mail: M.DAN HOF@LACDR.leidenuniv.nl)
}

for any potential PK interactions $(14,15)$. The characterization of PK in pre-clinical PD investigations is not without complications, as blood sampling may interfere with behavioral measurements. This may require the application of innovative study designs and data-analysis techniques (16-18).

Pregabalin exhibits its activity as a ligand to the $\alpha_{2} \delta$ subunit of the voltage gated calcium channel (VGCC). Avid binding at this site reduces calcium influx at nerve terminals and therefore reduces the release of several neurotransmitters including glutamate, norepinepherine and substance $\mathrm{P}$ (19). It has been demonstrated to exhibit anti-allodynic (20, $21)$, anxiolytic $(22,23)$ and anticonvulsant (24) activity.

Sildenafil is a potent and selective Phosphodiesterase type 5 (PDE-5) inhibitor. PDE-5 is an enzyme primarily responsible for the breakdown of cGMP, an intracellular second messenger. As the breakdown of cGMP is inhibited, increased intracellular concentrations of cGMP result and are thought to be primarily responsible for its therapeutic activity. It was originally investigated to treat angina, but its current therapeutic indication is in the treatment of male erectile dysfunction (25). Due to the prevalence of PDE-5 in many systems in the body, sildenafil has also been successfully applied for the treatment of other conditions, including pulmonary hypertension $(26,27)$. Recently, it has been observed that sildenafil enhances the effects of pregabalin in an animal model of neuropathic pain (28). While the 
mechanism of interaction is unknown, it is hypothesized that sildenafil may exhibit a PD synergistic interaction with pregabalin by increasing intracellular concentrations of cGMP. In turn, increased intracellular cGMP may amplify downstream regulation of the VGCC and modify pain perception in neuropathic pain.

The scope of this paper is to describe the development of a PK model identifying any potential PK interaction between pregabalin and sildenafil in rats with the objective of informing the design of a future quantitative PD study examining this interaction. Furthermore, the ultimate goal is the translation of this interaction to guide the first investigations of this drug combination in humans (29).

The overall process of informing the PD study design is outlined in Fig. 1. First, a PK study is conducted and a PK model developed using data collected from that study. This PK model provides the basis for investigating optimal PK sampling strategies for a PD study where PK sampling is not possible during the PD experiment. The necessity of designing the PK sampling in this way was demonstrated by a decrease in precision of PD endpoints during pilot studies where blood sampling was conducted in a chronic constriction injury rat model of neuropathic pain (Bender et al., unpublished). This distortion required that individual animal PK, ultimately for use in the PK-PD model, be estimated in animals without any blood samples taken during the PD portion of the experiment. To this end, a series of simulations was designed to evaluate the most parsimonious sampling strategy from among a series of eight sampling scenarios (17, 18, 30). The population approach to the PK study design allows the integrated application of PK samples from our dense PK study with the sparser sampling planned for our PD experiment. Through the application of nonlinear mixed effects modeling utilizing a Bayesian approach, the dense PK information can inform the PK model fit for those individuals with sparse sampling, allowing reconstruction of a full PK time course projected during the PD experiment $(15,31-34)$. Through examination of bias and precision of the

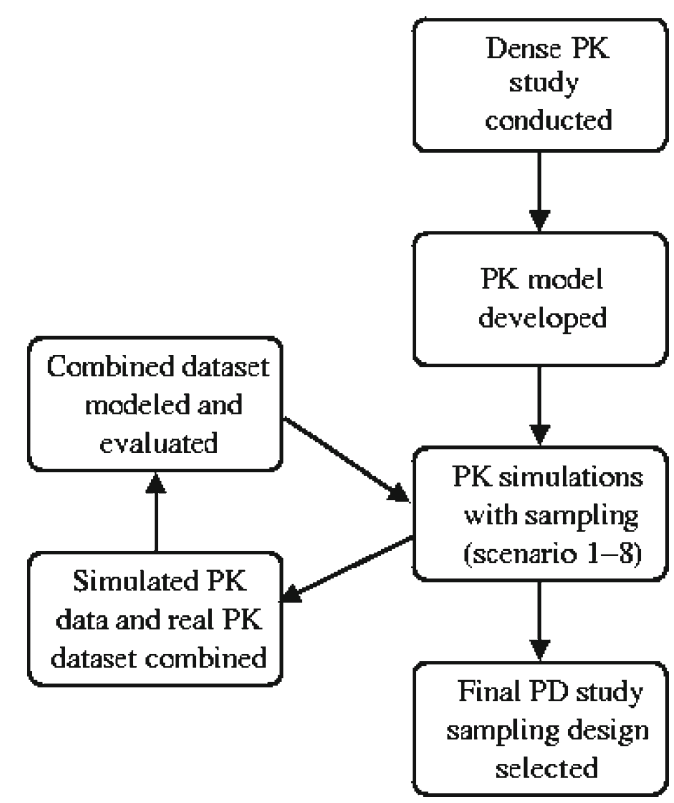

Fig. 1. Flowchart depicting $\mathrm{PK}$ modeling and the simulations conducted in this study. prediction errors for the PK and PD parameters from each of the simulated sampling scenarios, the most parsimonious sampling strategy was selected.

\section{MATERIALS AND METHODS}

\section{Chemicals}

Pregabalin, sildenafil and the N-methyl metabolite of sildenafil were obtained from the Compound Control Unit of Pfizer Global Research and Development, Pfizer UK.

\section{Animals}

Male Sprague-Dawley rats (200-250 g), obtained from Charles River Laboratories (Margate,UK), were housed under a 12:12 h light-dark cycle with food and water ad libitum.

\section{Surgical Procedures}

All experiments were conducted in a chronic constriction rat model of neuropathic pain as previously described by Bennett and Xie (35). Briefly, this surgical procedure involved tying four loose ligatures around the right sciatic nerve. After this procedure, the animal developed a peripheral mononeuropathy which resembles the human condition in its response to static and dynamic allodynia (35).

Jugular venous catheters (JVC) and carotid artery catheters (CAC) were implanted according to standard surgical procedures. Briefly, this entailed making a small incision in the skin over the right jugular vein or left carotid artery and blunt dissection through the subcutaneous fat and connective tissue to expose the vessel. A section of vessel approximately $5 \mathrm{~mm}$ in length was then isolated, a loose ligature was tied caudally and the cranial end was ligated. A small incision was made between these ligatures, and the catheter was introduced and secured in place with the preplaced sutures. The catheter was then tunnelled subcutaneously to exit through the scapula region into a button of a Dilab® automated blood sampling apparatus. A local ethics review committee approved all experimental protocols.

\section{Drug Administration and Blood Sampling}

During this investigation, the PK of pregabalin was determined in the presence of either a steady-state concentration of sildenafil, targeted at maximum target inhibition, or placebo. Drugs were administered as an intravenous infusion through the jugular venous catheter. Volumes and infusion rates were kept constant throughout all treatment groups. Sildenafil or placebo was administered by a combined bolus and continued infusion. Specifically, a $1 \mathrm{~mL} / \mathrm{kg}$ bolus containing $2 \mathrm{mg} / \mathrm{kg}$ sildenafil was administered over $10 \mathrm{mins}$ followed by an infusion rate of $2 \mathrm{~mL} / \mathrm{kg} / \mathrm{hr}$ containing $12 \mathrm{mg} / \mathrm{kg} / \mathrm{hr}$ of sildenafil for the duration of the planned $6 \mathrm{~h}$ PD experiment. Pregabalin was administered as a 2-hr infusion (doses of $10 \mathrm{mg} / \mathrm{kg} / \mathrm{hr}$ or $4 \mathrm{mg} / \mathrm{kg} / \mathrm{hr}$ ), as this was determined during pilot studies to be the optimal administration schedule. Specifically, this infusion length allowed characterization of the PD endpoints during both the upswing and downswing in concentration change during the course of the 6-hr PD sampling planned for the 
Table I. Study Design for the PK Investigations. Four Groups of Seven Rats each were Administered Either Pregabalin and Saline or Pregabalin and Sildenafil on Two Separate Days as Described Above

\begin{tabular}{lcc}
\hline & \multicolumn{1}{c}{ Day 1} & Day 4 \\
\hline Group 1 CCI rats pregabalin infusions with saline & \\
Group 1A 7 rats & $2 \mathrm{~h}$ pregabalin infusion at $4 \mathrm{mg} / \mathrm{kg} / \mathrm{hr}$ & 2 $\mathrm{h}$ pregabalin infusion at $10 \mathrm{mg} / \mathrm{kg} / \mathrm{hr}$ \\
& Saline loading dose and continuous infusion & Saline loading dose and continuous infusion \\
Group 1B 7 rats & $2 \mathrm{~h}$ pregabalin infusion at $10 \mathrm{mg} / \mathrm{kg} / \mathrm{hr}$ & $2 \mathrm{~h}$ pregabalin infusion at $4 \mathrm{mg} / \mathrm{kg} / \mathrm{hr}$ \\
& Saline loading dose and continuous infusion & Saline loading dose and continuous infusion \\
Group 2 CCI rats pregabalin infusions with sildenafil & \\
Group 2A 7 rats & $2 \mathrm{~h}$ pregabalin infusion at $4 \mathrm{mg} / \mathrm{kg} / \mathrm{hr}$ & $2 \mathrm{~h}$ pregabalin infusion at $10 \mathrm{mg} / \mathrm{kg} / \mathrm{hr}$ \\
& Sildenafil $2.2 \mathrm{mg}$ loading dose and $12 \mathrm{mg} / \mathrm{kg} / \mathrm{hr}$ & Sildenafil $2.2 \mathrm{mg}$ loading dose and $12 \mathrm{mg} / \mathrm{kg} / \mathrm{hr}$ \\
& continuous infusion & continuous infusion \\
Group 2B 7 rats & $2 \mathrm{~h}$ pregabalin infusion at $10 \mathrm{mg} / \mathrm{kg} / \mathrm{hr}$ & pregabalin infusion at $4 \mathrm{mg} / \mathrm{kg} / \mathrm{hr}$ \\
& Sildenafil $2.2 \mathrm{mg}$ loading dose and $12 \mathrm{mg} / \mathrm{kg} / \mathrm{hr}$ & Sildenafil $2.2 \mathrm{mg}$ loading dose and $12 \mathrm{mg} / \mathrm{kg} / \mathrm{hr}$ \\
& continuous infusion &
\end{tabular}

subsequent experiments. Table I outlines the dosages used in the development of the PK model.

Blood samples were obtained with the use of a Dilab ${ }^{\circledR}$ automated blood sampling apparatus. The Dilab® sampler obtained blood samples of $200 \mu \mathrm{L}$ through the carotid artery catheter into heparinized containers. After blood sampling, samples were centrifuged at 3,000 rpm for 3 mins, and plasma was separated for storage at $-20^{\circ} \mathrm{C}$ until analysis.

\section{Pharmacokinetic Study Design}

The PK study was designed as a crossover study on two occasions with a washout between occasions as outlined in Table I. A total of 28 rats divided evenly $(n=7)$ among four treatment groups were evaluated. The purpose of this multiday study was to allow independent estimation of the intraindividual and the intra-occasion variability as well as to conserve animals.

PK sampling was conducted in a staggered population approach using seven different sampling schedules corresponding to one rat from each of the four treatment groups (Table II). Up to eight PK samples were taken from each animal between $0.33-22 \mathrm{~h}$ after the start of the infusion on a particular experimental day (Table II). Each rat was sampled on day 1 and with at least a three-day washout period (day 4). An optimal sampling algorithm (D-optimal) was utilized as implemented in the ADAPT II software (36) to aid in the selection of these time points.

Table II. Sample Times for Individual Rats During the PK Study. Animal Number 1-7 Corresponds to Rat 1-7 in Each of the Four Treatment Groups

\begin{tabular}{cl}
\hline Animal number & \multicolumn{1}{c}{ Sample times (hrs) } \\
\hline 1 & $0.33,1.33,2.33,3.5,5.5,7.5,12,22$ \\
2 & $0.33,1.33,2.33,4.5,8.5,12.5,17,22$ \\
3 & $0.66,1.66,2.5,4,8,11.5,16,22$ \\
4 & $0.66,1.66,2.5,5,9,11,15,22$ \\
5 & $1,2,3,4.5,8,12,16,22$ \\
6 & $1,2,3,5,9,12.5,17,22$ \\
7 & $1,2,3.5,5.5,9.5,12.5,16,22$ \\
\hline
\end{tabular}

\section{Drug Analysis}

Plasma samples were analyzed for quantification of pregabalin, sildenafil and the major active N-methyl metabolite of sildenafil by an LC-MS/MS method briefly described below.

The extraction of pregabalin, sildenafil and the N-methyl metabolite was performed using solid phase extraction. Rat plasma samples, standards and quality controls $(50 \mu \mathrm{L})$ were mixed with $450 \mu \mathrm{L}$ water and then $500 \mu \mathrm{L} 1 \mathrm{M}$ mono chloroacetic acid in methanol/water $(1: 9 v / v)$ prior to extraction using activated $(2 \mathrm{ml}$ methanol) solid-phase extraction sorbent (Oasis MCX, Waters Corporation) packed in a 96 well format with $30 \mathrm{mg}$ sorbent per $2 \mathrm{ml}$ well. Following sample application, the cartridges were washed sequentially with $1 \mathrm{ml}$ water followed by $1 \mathrm{ml}$ of $1 \mathrm{M}$ mono chloroacetic acid in methanol/water $(1: 9 \mathrm{v} / \mathrm{v})$ followed by $1 \mathrm{ml}$ methanol. Pregabalin, sildenafil and the N-methyl metabolite and internal standards (UK-464,243 and UK-343,664) were eluted with $1 \mathrm{ml}$ of ammonium hydroxide:methanol $(5: 95 \mathrm{v} / \mathrm{v})$ into $2 \mathrm{ml}$ deep, 96 well blocks. These extracts were retained in the blocks, evaporated to dryness under a nitrogen stream $\left(37^{\circ} \mathrm{C}\right.$ ) and resuspended in $200 \mu \mathrm{L} 2 \mathrm{mM}$ ammonium acetate, $\mathrm{pH} 4.0$, in methanol water $(10: 90 \mathrm{v} / \mathrm{v})$.

Samples were analyzed on a Sciex API 3,000 mass spectrometer in positive mode using turbo ionspray. A fast gradient HPLC was used with a Chromolith Speed Rod RP-18e, $50 \times 4.6 \mathrm{~mm}, 5 \mu \mathrm{m}$ (Merck) at $3 \mathrm{ml} / \mathrm{min}$ with flow splitting ratio 1 to 5. Mobile phases of $0.027 \% \mathrm{HCOOH} v / v+10 \mathrm{mM}$ ammonium acetate $90: 10$ water: $\mathrm{CH}_{3} \mathrm{OH}$ (organic mobile phase) and $0.027 \% \mathrm{HCOOH} v / v+10 \mathrm{mM}$ ammonium acetate 90:10 $\mathrm{CH}_{3} \mathrm{OH}$ :water (aqueous mobile phase) were used and prepared fresh on each day. The method scanned for all compounds simultaneously. The MRM transitions used for each compound were as follows: pregabalin (160 to 142); UK-464,242 (184 to 107); sildenafil (475 to 58); the N-methyl metabolite of sildenafil (461 to 283); and UK-343,664 (566 to 346).

Intra- and inter-day assay accuracy and precision were assessed for each compound at 150, 2,000, 9,000 ng/ml for pregabalin and $3,500,1,800 \mathrm{ng} / \mathrm{ml}$ for sildenafil and UK103,302 spanning the calibration range $(n=5)$. Accuracy was assessed based upon the percentage of the ratio of the found concentration on the nominal concentration. Intra-day assay accuracies ranged from $95.5 \%$ to $107.6 \%$ for pregabalin, 
$101.2 \%$ to $111.1 \%$ for sildenafil and $99.8 \%$ to $107.2 \%$ for $\mathrm{N}-$ methyl metabolite of sildenafil. Inter-day (3 days) assay accuracies ranged from $101.2 \%$ to $108.3 \%$ for pregabalin, $101.2 \%$ to $108.3 \%$ for sildenafil and $100.0 \%$ to $102.8 \%$ for UK-103,320. Precision of the assay was expressed as \%CV (coefficient of variability) calculated from five replicates at three concentrations. \%CV for intra-day assay was 10.1 (150 ng/ml), $7.3(2,000 \mathrm{ng} / \mathrm{ml}), 5.5(9,000 \mathrm{ng} / \mathrm{ml})$ for pregabalin, 10.7 (3 ng/ml), $5.1(500 \mathrm{ng} / \mathrm{ml}), 9.6(1,800 \mathrm{ng} / \mathrm{ml})$ for sildenafil, and $11.3(3 \mathrm{ng} / \mathrm{ml}), 5.4(500 \mathrm{ng} / \mathrm{ml}), 8.4$ $(1,800 \mathrm{ng} / \mathrm{ml})$ for $\mathrm{UK}-103,320$. \% CV for inter-day assay was $13.2(150 \mathrm{ng} / \mathrm{ml}), 10.2(2,000 \mathrm{ng} / \mathrm{ml}), 11.6(9,000 \mathrm{ng} / \mathrm{ml})$ for pregabalin, $14.5(3 \mathrm{ng} / \mathrm{ml}), 5.3(500 \mathrm{ng} / \mathrm{ml}), 7.8(1,800 \mathrm{ng} / \mathrm{ml})$ for sildenafil, and $13.8(3 \mathrm{ng} / \mathrm{ml}), 6.6(500 \mathrm{ng} / \mathrm{ml}), 9.0$ $(1,800 \mathrm{ng} / \mathrm{ml})$ for UK-103,320. Quantitation was linear over the following concentration ranges: pregabalin (100-10,000 ng/ $\mathrm{ml})$, sildenafil $(3-2,000 \mathrm{ng} / \mathrm{ml})$ and the $\mathrm{N}$-methyl metabolite of sildenafil $(5-2,000 \mathrm{ng} / \mathrm{ml})$. The lower limit of quantitation for pregabalin, sildenafil, and the N-methyl metabolite of sildenafil was $70 \mathrm{ng} / \mathrm{ml}, 1 \mathrm{ng} / \mathrm{ml}$, and $1 \mathrm{ng} / \mathrm{ml}$, respectively.

\section{Data Analysis}

All PK modeling was accomplished utilizing a standard nonlinear mixed effects approach implemented within NONMEM (double precision Version 5, level 1.1, GloboMax, Hanover, MD) $(37,38)$. The models consisted of a structural model that described the disposition of the drug following IV administration, and a pharmacostatistical model that described the between-subject, between-occasion and residual variability. Diagnostic graphics, exploratory analyses, and post-processing of NONMEM outputs were performed using S-PLUS (Version 7.0 for windows, Insightful, Seattle, WA) with a NONMEM interface developed by LAP\&P consultants BV (Leiden, the Netherlands).

The first order conditional estimation method (FOCEINTER) was used for model building. The adequacy of each model was assessed using standard goodness-of-fit plots, the precision of the parameter estimates (as calculated using the COVARIANCE option of NONMEM) and the NONMEM objective function value (OFV). When discriminating between hierarchical models based on OFV, the likelihood ratio test was applied. This test is based on the property that the ratio of the NONMEM objective function values (OFV) (-2 log-likelihood) were asymptotically $\chi^{2}$ distributed. An objective function decrease of 10.8 units was considered significant $\left(\chi^{2} p<0.001 \mathrm{df}=1\right)$.

Standard errors for all parameters were obtained using the covariance option in NONMEM. The population PK analysis was performed using the NONMEM subroutine ADVAN 3 TRANS 4 (two-compartment model). It was assumed that both the between-occasion variability (BOV) and the betweensubject variability (BSV) of the PK parameters were lognormally distributed. Therefore, the relationship between a PK parameter $(P)$ and its variance was expressed as:

$$
P_{i}=P_{T V} \times e^{\left(\eta B S V_{P}+\eta B O V_{P}\right)}
$$

Here, $P_{i}$ was the value of PK parameter for the $i^{\text {th }}$ individual, $P_{T V}$ was the typical value of $P$ for the population, and $\left(\eta B S V_{P+} \eta B O V_{P}\right)$ denoted the difference between $P_{i}$ and $P_{T V}$, independently, which was identically distributed with a mean of zero and variance of $\omega_{\mathrm{P}}{ }^{2} . \eta B S V$ refers to the between-subject variability, while $\eta B O V$ is the between-occasion variability.

The residual variability was examined using additive, proportional, and combined error structures as described below:

$$
\begin{gathered}
\text { Additive error : } y_{i j}=\widehat{y}_{i j}+\varepsilon_{i j} \\
\text { Proportional error : } y_{i j}=\widehat{y}_{i j}\left(1+\varepsilon_{i j}\right) \\
\text { Combined additive and proportional error : } \\
\qquad y_{i j}=\widehat{y}_{i j}\left(1+\varepsilon_{i j}\right)+\varepsilon_{i j}{ }^{\prime}
\end{gathered}
$$

Here, $y_{i j}$ was the $j^{\text {th }}$ observation in the $i^{\text {th }}$ individual, $\hat{y}_{i j}$ was the corresponding model prediction, and $\varepsilon_{i j}$ (or $\varepsilon_{i j}^{\prime}$ ) was a normally distributed random error with a mean of zero and a variance of $\sigma^{2}$.

The final model was developed by testing the effect of subject-specific covariates' bodyweight, age, time post CCI-surgery, time post catheterization surgery, sildenafil concentration, and sildenafil's metabolite concentration. All covariates were initially modeled as continuous. Sildenafil was also modeled as a discrete covariate as the steady state infusions used during the PK study resulted in a relatively stable concentration of sildenafil which would saturate its target over the experimental period of interest. Stepwise covariate selection was utilized for the covariate modelbuilding (39-43). First, exploratory covariate selection was performed by examination of the normalized eta deviation between individual post-hoc parameter estimates and candidate covariates. Subsequently, various forms of parameterization of the selected covariates were added to the base model and evaluated for significance by observing $\triangle \mathrm{OFV}$ and diagnostic plots. Only the single covariate parameterization producing the most significant increase in goodness of fit then moved on to the next stage. This continued until no significant improvements in model fit could be gained through further covariate inclusion.

The following example shows the effect of a continuous covariate on $C L$ :

$$
\begin{gathered}
T V C L=\theta_{C L} \times\left(\operatorname{Cov} / \operatorname{Med}_{C o v}\right)^{\theta_{C o v}} \\
C L=T V C L \times \exp \left(\eta_{i}\right)
\end{gathered}
$$

$T V C L$ was the typical value for the population; $\eta_{i}$ was the random effect representing the difference of the $i^{\text {th }}$ subject from the population mean. The random effects of between-subject variability were assumed log-normally distributed, with a mean of zero and standard deviation of $\omega$. Cov was the continuous covariate that was affecting $C L$, and $M e d_{C o v}$ was the median Cov. The following example shows the effect of a discrete covariate sildenafil presence (SLDB) on $C L$ :

$$
T V C L=\theta_{C L}+(1-S L D B) \times \theta_{S L D}
$$

When $S L D B$ was 0 (sildenafil absence $=0$, sildenafil presence $=1), T V C L$ equals $\theta_{C L}$ and when $S L D B$ was 1 , the 
$\theta_{S L D}$ term was subtracted from the population estimate of $C L$. The final model was evaluated utilizing bootstrapping with resampling technique to examine the stability of the final model and estimate confidence intervals of the parameters (44-46). This technique consists of fitting the model to 100 bootstrapped replicates of the dataset using the bootstrap option in the LAP\&P software interface for SPLUS. Parameter estimates for each of the replicate data sets were obtained. The results from 500 successful runs were obtained, and the mean and 2.5th and 97.5th percentiles (denoting the $95 \%$ confidence interval) for the population parameters were determined and compared with the estimates of the original data.

A posterior predictive check was performed (47). A posterior predictive check involves utilizing the simulation option within NONMEM to create $90 \%$ prediction bands. These are obtained by simulating 1,000 replications using the final model and a dataset including identical experimental design. For each observation, the values of the 51st highest and the 51st lowest prediction become the edges of the $90 \%$ prediction band. Observed values are then plotted on the same scale as these bands to help visualize the adequacy of model fit. After completion of these steps, the final descriptive PK-PD model was proposed.

\section{Simulations to Evaluate Pharmacokinetic Sampling Paradigms}

The population PK model was used as the basis of simulations designed to identify the most parsimonious PK sampling schedule for implementation in the future PK-PD study. The simulation study design was selected to mimic the pregabalin-only portion of the planned PD study. Doses and number of animals per group were previously determined from pilot experiments based on the nature of the PD interaction and what could be realistically utilized in these types of experiments, respectively. As such, the motivation for the simulation PK study was intended only to optimize the PK sampling portion of future PD experiments.

PK profiles for pregabalin doses of 4 and $10 \mathrm{mg} / \mathrm{kg} / \mathrm{hr}$ were simulated with 12 rats in each dose group for each sampling scenario. Simulated samples were generated from these profiles according to the sampling schedule. A total of eight sampling scenarios were examined as outlined in Table III. Next, the simulated samples were combined back with the real-world PK samples to serve as an anchor for the

Table III. Sampling Scenarios and the Corresponding Sampling Times Investigated for Proposed Downstream PK-PD Studies. PD Sampling Duration was Treated as Lasting $6 \mathrm{~h}$ Following the Start of a 2-hr Pregabalin Administration

\begin{tabular}{cl}
\hline Sampling scenario & \multicolumn{1}{c}{ Sample times $(\mathrm{hr})$} \\
\hline 1 & 6 \\
2 & 6,8 \\
3 & $6,8,24$ \\
4 & $6,8,10,12,15,18,21,24$ \\
5 & $1,2,4,6,8,12,18,24$ (another occasion) \\
6 & $1,2,4,6,8,12,18,24$ \\
7 & 2,6 \\
8 & No sampling \\
\hline
\end{tabular}

population PK model. These new datasets were then remodeled, and the newly predicted individual PK parameters were compared with those used to create the simulation. Prediction errors were then calculated to determine the bias and precision of predictions provided through each sampling scenario. After this evaluation process was completed for the PK model, a PD model examining the impact of the various PK sampling schedules on the ability to predict PD was evaluated in a similar fashion. More detailed methods are described below.

Sampling scenarios to be evaluated (Table III) were selected to compare various post-PD sampling schedules with the best case (eight PK samples) and worst case (no samples) scenarios. In addition, a sampling design with only a single PK sample taken during the PD portion of the experiment was simulated. The first four scenarios included only post-PD sampling. The fifth scenario examined the effects of dense sampling during the entire experiment, but on another occasion. The sixth schedule examined the hypothetical best-case scenario of dense sampling during the entire experiment (were it possible). The seventh scenario examined the potential benefit of including one sample during the PD experiment and one immediately after. Finally, the worst case scenario of obtaining no individual animal PK was assessed.

Monte-Carlo simulations were used to examine the impact of the various sampling schedules on the identification of the individual animal-specific PK. In this procedure the simulation function of NONMEM creates "simulated rats." These simulated rats have PK parameters (simulated parameters) selected from within the distribution of possible parameters defined by the final PK model with the assistance of a random number generator. Simulated PK profiles were then generated for each of the simulated rats with samples taken from each of these profiles according to the proposed sampling schedule. These simulated concentration points were then combined with the complete PK dataset (real dataset from the dense PK study), along with the "true" individual-specific simulated parameter estimates (for future comparison). This new simulated dataset was modeled in NONMEM utilizing the same methods as described in the PK analysis section generating predicted parameters. This whole process is outlined in Fig. 1.

This cycle of simulation and estimation was repeated 100 times for each of the eight sampling schedules creating 100 "simulated experiments" for each. Model performance was then assessed examining how close the predicted parameters were to the "true" or simulated parameters using the prediction error on the individual clearance parameter estimates. The prediction error $(P E)$ was calculated as follows:

$$
P E=\frac{\theta_{\text {pred }}-\theta_{\text {sim }}}{\theta_{\text {sim }}} \times 100 \%
$$

The prediction errors were visualized for each sampling schedule using boxplots to examine their bias (median prediction error) as well as precision (quartiles) for each replication. The bias and precision along with practical considerations served to guide the sampling protocol selection.

A previously developed PK-PD model was then applied to examine the ability to accurately identify the EC50 for static allodynia given three sampling scenarios (best(6), worst 
(8), and chosen(3)). In this PK-PD model, the PK approach described above was applied. This was coupled with an effect comparment, which was used to drive the PD model. The PD model consisted of a sigmoid EMAX model to relate the concentration of drug available at the effect site $(C e(t))$ to the observed response expressed as a percentage change in response to static allodynia from baseline. In this model, $E M A X$ is the maximum change in response the drug can produce (fixed to 1 or $100 \%$ ), EC50 is the value of $\mathrm{Ce}(t)$ producing $50 \%$ of the EMAX value, and Hill influences the steepness of the relationship:

$$
E(t)=\frac{E M A X \cdot C e(t)^{\text {Hill }}}{E C 50^{H i l l}+C e(t)^{H i l l}}
$$

Parameters for the Emax model were obtained from a pilot study in a chronic constriction injury model of neuropathic pain, using the difference in paw withdrawal threshold upon stimulation with von Frey hairs as a pharmacodynamic endpoint (28). The study was conducted in male Sprague Dawley rats (Charles River Laboratories (Margate,UK). The values of the (population) pharmacodynamic parameters were $\mathrm{Keo}=6.27 \mathrm{~h}(23.6 \%)$, EC50 $=9.36 \mathrm{ug} / \mathrm{mL}(5.3 \%)$, Emax $=1$, and Hill $=3.8(8.3 \%)$, assuming a normal distribution. PD parameters and plots were simulated and re-evaluated in NONMEM in the same way as the PK simulations. From the simulated and predicted PD parameters, prediction errors for EC50 were calculated and evaluated in the same way as the PK parameters and considered in the selection of the most appropriate sampling strategy.

\section{RESULTS}

Concentrations of pregabalin, sildenafil and the active hydroxyl metabolite of sildenafil are displayed in Fig. 2. Maximal concentrations of pregabalin were reached at the end of the 2-hr infusion and are roughly 22,000 and 10,000 ng/ $\mathrm{mL}$ respectively for the $10 \mathrm{mg} / \mathrm{kg} / \mathrm{hr}$ and $4 \mathrm{mg} / \mathrm{kg} / \mathrm{hr}$ pregabalin infusions. When sildenafil was administered concomitantly, visible increases in the concentrations of pregabalin can be observed.

A two-compartment model was chosen as the final structural model to describe the concentration time course of pregabalin. Estimated model parameters are listed in Table IV. Both between-subject variability and betweenoccasion variability were determined in the base model for clearance (BSV 0.024(24.7\%), BOV 0.098(27.1\%)) and the volume of the central compartment (BSV 0.017(9.4\%), BOV $0.015(9.0 \%)$ ). A proportional error model was selected to account for the residual variability. Overall diagnostic plots demonstrated a good model fit (Fig. 3). Observed values versus both population and individual predicted were near the line of identity. Weighted residuals were relatively small in magnitude and randomly distributed when viewed versus time and concentration.

Of all the covariates investigated, only sildenafil and its metabolite had a significant impact on the model. This is likely due to the homogeneous nature of the inbred rat strain and controlled conditions of the experiment. Two final covariate models incorporating the presence of sildenafil were decided upon. First, a binary model was implemented
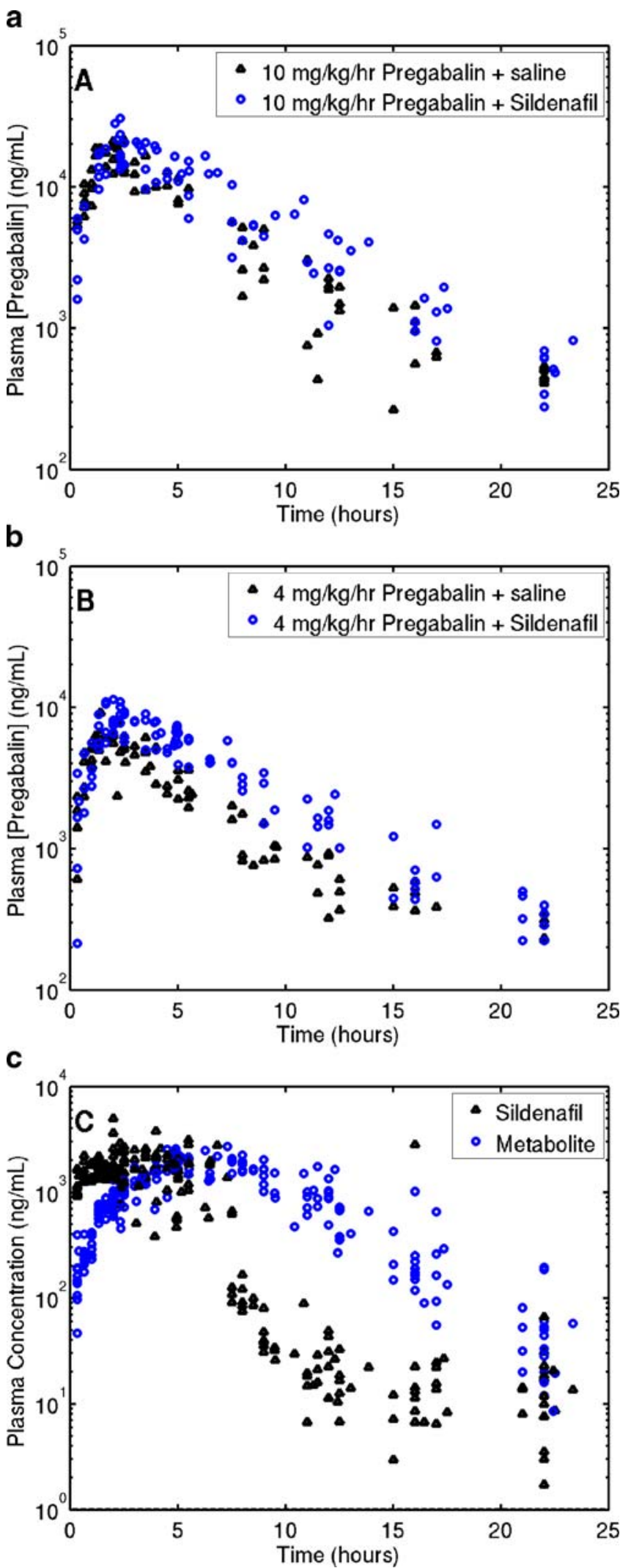

Fig. 2. Raw data concentration $v$ s. time plots for: $\mathbf{A}$ a 2-h infusion of pregabalin at $10 \mathrm{mg} / \mathrm{kg} / \mathrm{hr}+$ saline, $\mathbf{B}$ for a $2-\mathrm{hr}$ infusion of pregabalin at $4 \mathrm{mg} / \mathrm{kg} / \mathrm{hr}+$ saline and $\mathbf{C}$ sildenafil and its active hydroxy metabolite from Group 2 rats. 
Table IV. Objective Function Values (OFV) and Parameter Value Estimates for the Two-Compartment Pregabalin PK Model. Sildenafil Interaction was Incorporated as Either a Binary $(1-\mathrm{SLDB}) \theta_{\mathrm{SLD}}$ or Continuous $\left(1-[\mathrm{SLDM}] /\left(\theta_{\mathrm{SLD}}+[\mathrm{SLDM}]\right)\right)$ Covariate Effect on Clearance. $\omega_{1}$ and $\omega_{2}$ Denote Between-Subject and Between-Occasion Variability, Respectively, for the Listed Model Parameter

\begin{tabular}{|c|c|c|c|c|c|c|c|c|c|c|c|c|}
\hline \multirow{3}{*}{ OFV } & \multicolumn{4}{|c|}{ Original PK Model } & \multicolumn{4}{|c|}{ Binary Sildenafil Covariate } & \multicolumn{4}{|c|}{ Continuous Sildenafil Metabolite Covariate } \\
\hline & \multicolumn{4}{|c|}{5405.6} & \multicolumn{4}{|c|}{5397.1} & \multicolumn{4}{|c|}{5363.0} \\
\hline & Value & $\mathrm{CV} \%$ & LLCI & ULCI & Value & $\mathrm{CV} \%$ & LLCI & ULCI & Value & $\mathrm{CV} \%$ & LLCI & ULCI \\
\hline$\overline{\mathrm{CL}}(\mathrm{L} / \mathrm{hr})$ & 0.034 & $19.08 \%$ & 0.021 & 0.046 & 0.052 & $14.59 \%$ & 0.037 & 0.066 & 0.051 & $8.83 \%$ & 0.042 & 0.060 \\
\hline $\mathrm{V} 1(\mathrm{~L})$ & 0.270 & $3.38 \%$ & 0.252 & 0.288 & 0.271 & $3.38 \%$ & 0.253 & 0.289 & 0.272 & $3.27 \%$ & 0.255 & 0.289 \\
\hline $\mathrm{Q}(\mathrm{L} / \mathrm{hr})$ & 0.0225 & $32.00 \%$ & 0.0084 & 0.0366 & 0.0151 & $35.76 \%$ & 0.0045 & 0.0257 & 0.0229 & $18.08 \%$ & 0.0148 & 0.0310 \\
\hline V2 (L) & 6.75 & $41.48 \%$ & 1.26 & 12.24 & 2.77 & $51.99 \%$ & -0.05 & 5.59 & 2.45 & $38.82 \%$ & 0.59 & 4.31 \\
\hline$\omega_{1} \mathrm{CL}^{\prime}$ & 0.024 & $24.74 \%$ & 0.012 & 0.035 & 0.017 & $15.91 \%$ & 0.012 & 0.022 & 0.013 & $14.63 \%$ & 0.009 & 0.016 \\
\hline$\omega_{1} \mathrm{~V} 1$ & 0.017 & $9.38 \%$ & 0.014 & 0.020 & 0.018 & $9.26 \%$ & 0.014 & 0.021 & 0.016 & $8.72 \%$ & 0.013 & 0.018 \\
\hline$\omega_{1} \mathrm{Q}$ & 0.430 & $40.00 \%$ & 0.093 & 0.767 & 0.439 & $40.87 \%$ & 0.087 & 0.791 & 0.081 & $15.94 \%$ & 0.055 & 0.106 \\
\hline$\omega_{2} \mathrm{CL}$ & 0.098 & $27.07 \%$ & 0.046 & 0.151 & 0.061 & $19.52 \%$ & 0.038 & 0.085 & 0.071 & $18.52 \%$ & 0.045 & 0.097 \\
\hline$\omega_{2} \mathrm{~V} 1$ & 0.015 & $8.96 \%$ & 0.013 & 0.018 & 0.015 & $8.75 \%$ & 0.012 & 0.017 & 0.016 & $8.33 \%$ & 0.013 & 0.019 \\
\hline$\theta_{\mathrm{SLD}}$ & - & - & - & - & 0.302 & $23.05 \%$ & 0.166 & 0.438 & $\begin{array}{l}1350 \\
(\mathrm{ng} / \mathrm{mL})\end{array}$ & $28.37 \%$ & 599 & 2101 \\
\hline oresidual & 0.029 & $18.32 \%$ & 0.019 & 0.040 & 0.029 & $18.53 \%$ & 0.019 & 0.040 & 0.029 & $18.85 \%$ & 0.018 & 0.039 \\
\hline
\end{tabular}
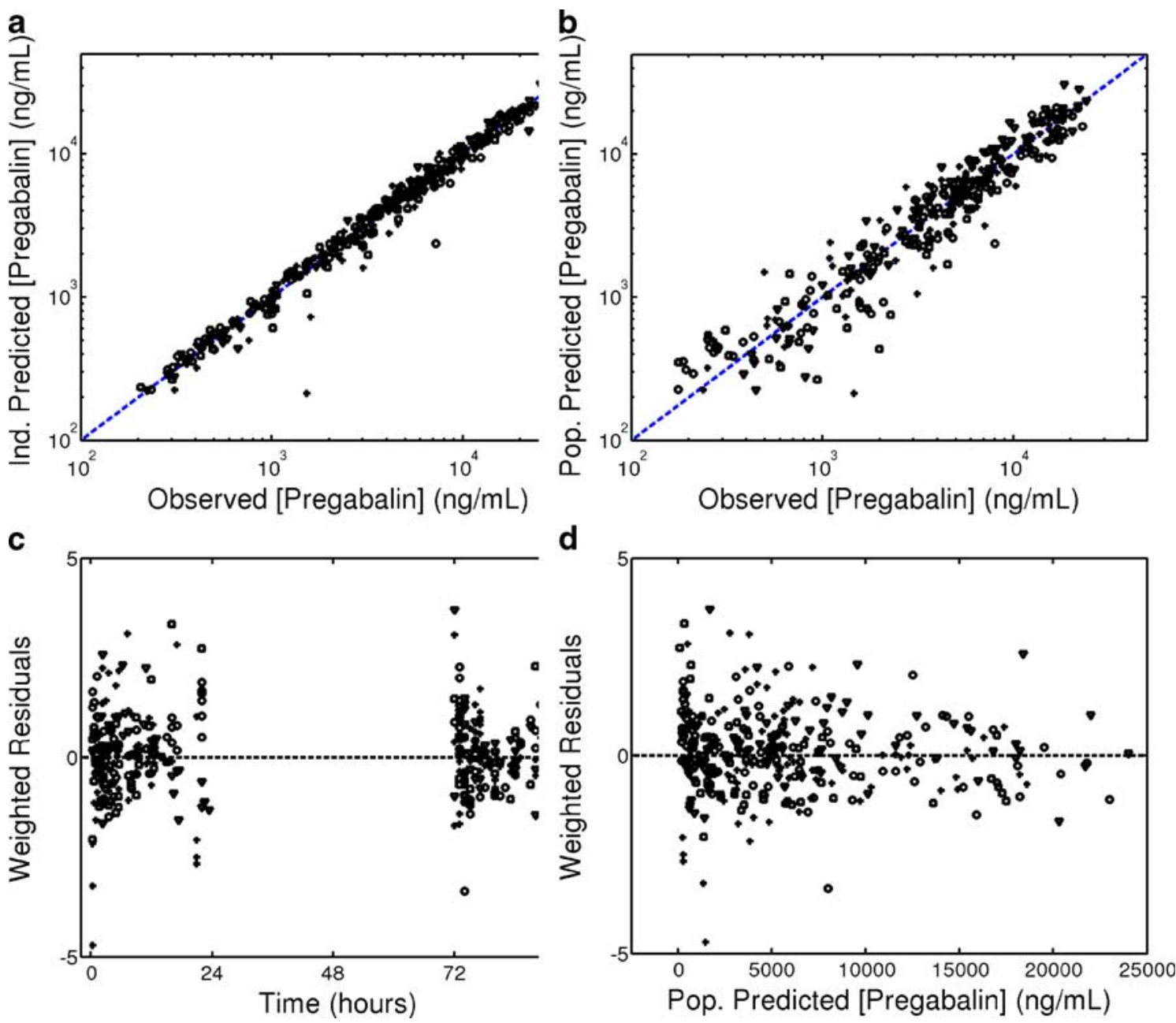

Fig. 3. Diagnostic plots for the pregabalin PK model: A observed $v$ s. individual predicted, $\mathbf{B}$ observed $v s$. population predicted, $\mathbf{C}$ weighted residuals vs. time, $\mathbf{D}$ and weighted residuals $v s$. predicted pregabalin concentration. Symbols depict data points from rats administered $4 \mathrm{mg} / \mathrm{kg}$ without sildenafil (circles), $10 \mathrm{mg} / \mathrm{kg}$ without sildenafil (squares), $4 \mathrm{mg} / \mathrm{kg}$ with sildenafil (+), and $10 \mathrm{mg} / \mathrm{kg}$ with sildenafil (triangles). 
examining the presence or absence of sildenafil in the experiment. The final parameters for this model are shown in Table IV. The effect on clearance was demonstrated graphically in Fig. 4. Sildenafil resulted in a reduction in pregabalin clearance of $30.2 \%$ with a $\mathrm{CV}$ of $23.1 \%$. The optimal descriptive model fit utilizing the continuous concentrations of sildenafil and its metabolite was also sought. Various parameterizations of the effect of sildenafil, sildenafil's metabolite, and combinations of the two were examined. The parameterization identified as giving the largest increase in goodness of fit was a nonlinear function utilizing only the plasma concentration of sildenafil's active metabolite $[S L D M]$ :

$$
T V C L=\theta_{C L}\left(1-\frac{[S L D M]}{\theta_{S L D}+[S L D M]}\right)
$$

Final parameter values for this model are listed in Table IV. Bootstrapping with replacement demonstrated a good model fit, mean parameter values remained near the final model, and CV\% was low. A posterior predictive check also demonstrated a good model fit. Fig. 5 demonstrates that most of the observed data points fall within the $90 \%$ prediction interval.

The resulting box-plot summaries for the PK-PD simulation prediction errors on clearance and EC50 are illustrated in Figs. 6 and 7, respectively. The first four sampling scenarios with post-PD sampling $(6 \mathrm{~h}$ or later) demonstrated that improved predictability of clearance occurred when more post-PD samples were obtained. Notably, improvements in both bias and precision were seen as the number of samples increased. However, sampling scenario 4 with eight PK samples was only slightly better than scenario 3 with only three post-PD samples. Sampling on another occasion, even with eight samples spaced throughout the experiment at equivalent times to scenario 5, was no better than obtaining no samples at all (comparable bias and precision for scenarios 5 and 8). Scenario 6 represented the hypothetical best-case situation if dense blood samples were taken throughout the experiment. The bias was negligible and the precision was the tightest for this scenario; however, the bias was comparable and the precision only slightly better than both scenarios 3 and 4. Adding a single sample during the PD portion of the experiment $(0-6 \mathrm{~h})$ in scenario 7 was of no added value in comparison with scenario 2 where two samples were taken after the PD experiment. Finally, scenario 8 served as a point of comparison where no PK samples were taken. As expected, this produced the most imprecise estimates and introduced the largest amount of prediction error, along with scenario 5 where blood samples were taken in the same animal, but on a separate occasion. There was no bias in this schedule as the typical parameter values were assumed for all individuals. PD simulations reflected these conclusions for the three simulated sampling schedules.

\section{DISCUSSION}

The development of a PK model identifying a PK interaction between pregabalin and sildenafil in rats was investigated. Furthermore, these results served to inform the design of a future quantitative PD study examining the interaction of these drugs. This PK study design also serves as the first step toward identifying and quantifying a synergistic PD interaction between pregabalin and sildenafil.

Fundamental to identifying the source for an observed interaction between two compounds is to take the influence of a potential PK interaction into account $(14,15)$. The results of this analysis served that purpose by quantifying the PK of pregabalin with and without the presence of sildenafil in rats, noting a $30.2 \%$ decrease in the clearance of pregabalin when coadministered with sildenafil. This decrease in clearance was unexpected, and the mechanism for the PK interaction remains unclear. One potential hypothesis to explain this interaction may be an effect of sildenafil on renal transporters affecting pregabalin, although there is no information to enlighten this theory. The decrease in pregabalin clearance did produce a slight increase in the observed plasma concentrations of pregabalin over the observation period of interest.

The effect of pregabalin on the PK of sildenafil was not investigated. $\mathrm{N}$-methyl sildenafil attained a $\mathrm{C}_{\max }$ of $2,100 \mathrm{ng} /$ $\mathrm{mL}$ at $4-7 \mathrm{~h}$, compared to sildenafil with a $\mathrm{C}_{\max }$ of $2,400 \mathrm{ng} /$ $\mathrm{mL}$ at $2-4 \mathrm{~h}$. While the metabolite $\mathrm{C}_{\max }$ was slightly lower
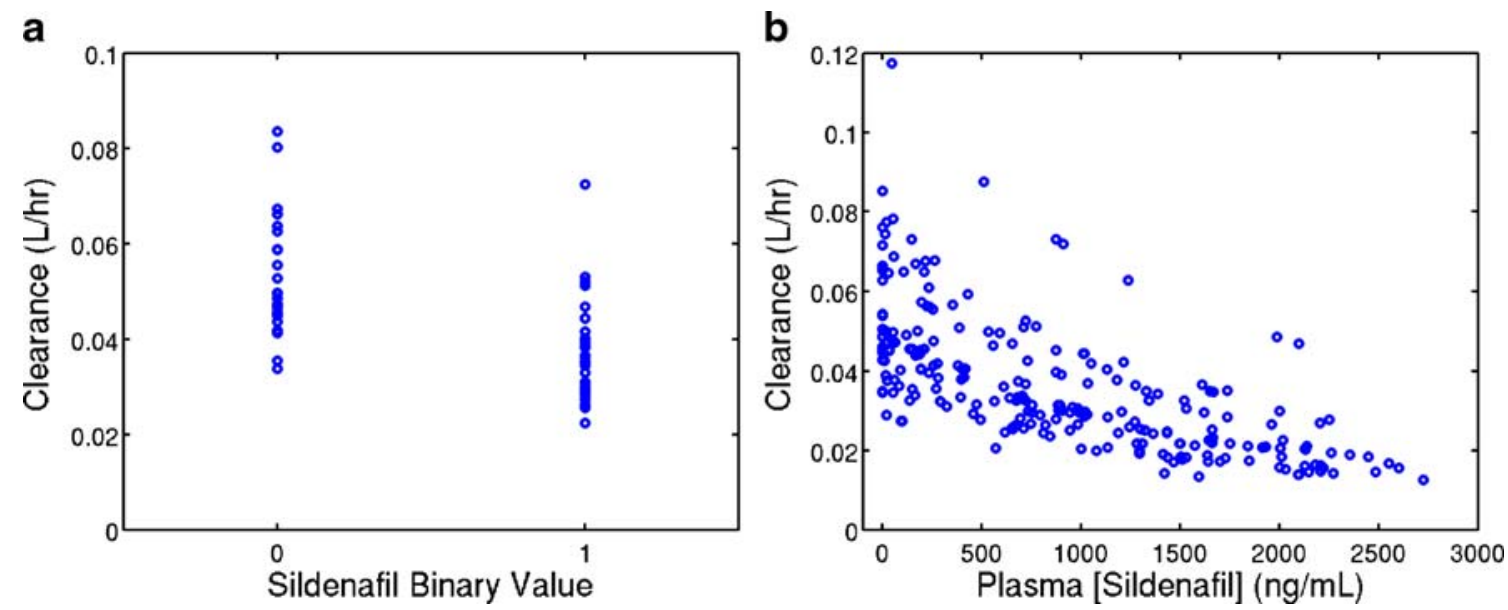

Fig. 4. Scatter plots demonstrating the effect of sildenafil administration on clearance: A pregabalin clearance vs. a binary variable representing either no sildenafil (0) or with sildenafil (1) and $\mathbf{B}$ pregabalin clearance $v s$. the concentration of sildenafil's metabolite. 


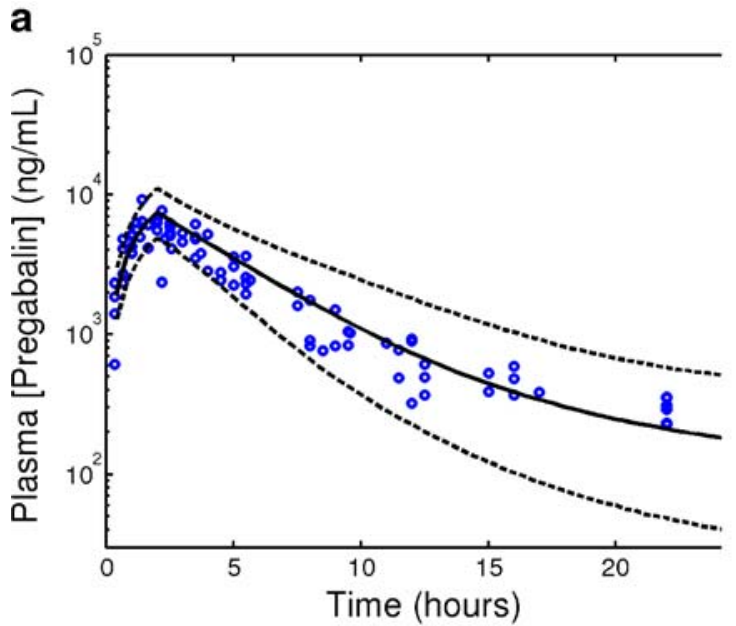

C

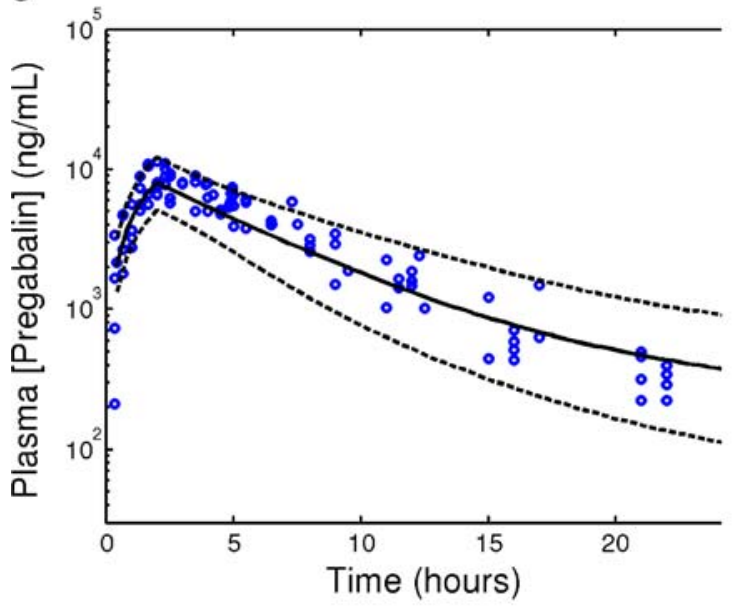

b

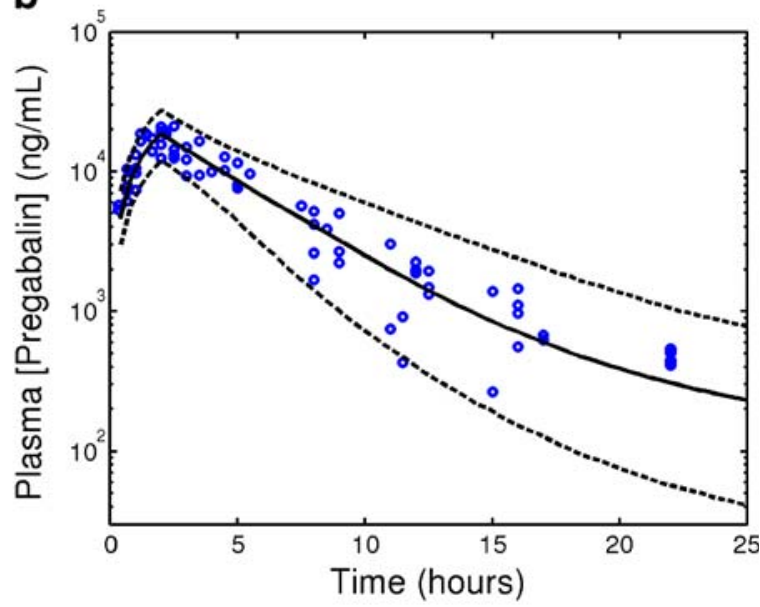

d

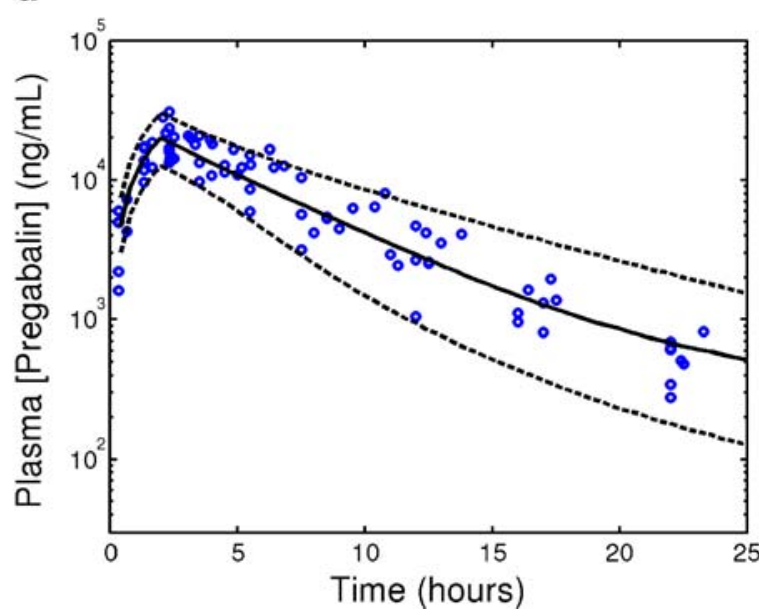

Fig. 5. Posterior predictive check of pregabalin concentration $v s$. time profiles for each of the treatment groups administered a 2-hr pregabalin infusion at: A $4 \mathrm{mg} / \mathrm{kg} / \mathrm{hr}$ without sildenafil, B $10 \mathrm{mg} / \mathrm{kg} / \mathrm{hr}$ without sildenafil, C $4 \mathrm{mg} / \mathrm{kg} /$ $\mathrm{hr}$ with sildenafil, and $\mathbf{D} 10 \mathrm{mg} / \mathrm{kg} / \mathrm{hr}$ with sildenafil. Lines represent the $90 \%$ prediction interval for the model.

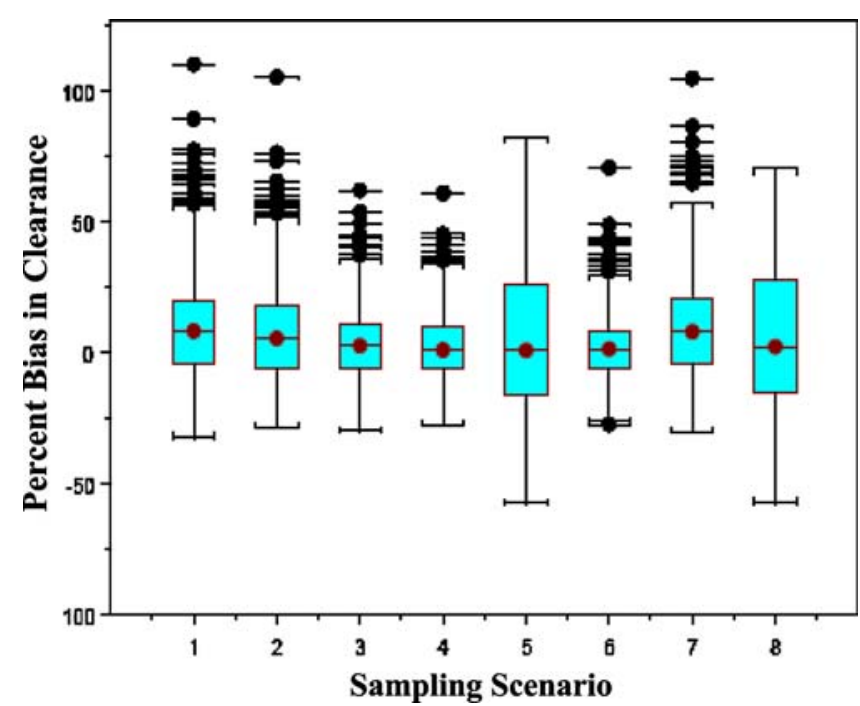

Fig. 6. Box plot summaries of prediction errors on clearance for each of the eight sampling schedule scenarios: 1. $(6 \mathrm{~h}), 2$. (6 and $8 \mathrm{~h}), 3$. (6, 8 , and $24 \mathrm{~h}), 4 .(6,8,10,12,15,18,21$, and $24 \mathrm{~h}), 5$. (another occasion: $1,2,4,6,8,12,18$, and $24 \mathrm{~h}), 6 .(1,2,4,6,8,12,18$, and $24 \mathrm{~h}), 7$. (2 and $6 \mathrm{~h}$ ), and 8 . no PK samples.

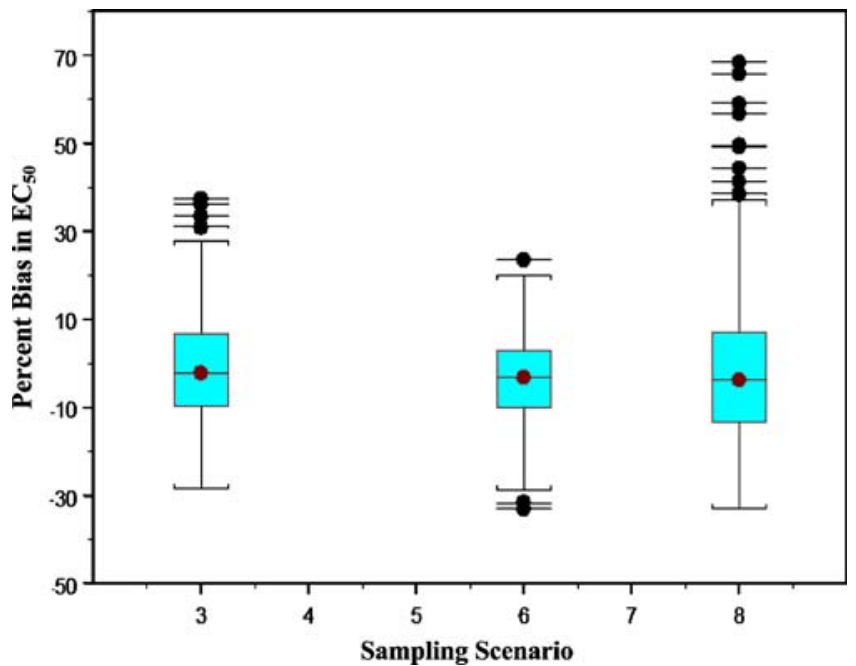

Fig. 7. Box plot summaries of prediction errors on $\mathrm{EC}_{50}$ for three of the eight sampling schedule scenarios: 3. (6, 8, and $24 \mathrm{~h}), 6$. (1, 2, 4, 6, $8,12,18$, and $24 \mathrm{~h}$ ), and 8 . no PK samples. 
than sildenafil, the ratio between metabolite/parent $(0.90)$ was much higher than that observed for the metabolite and parent in humans (0.4) (48). While the higher ratio may influence model development and subsequent human scaleup for non-saturating doses of sildenafil, the bolus and maintenance dose selected within this study attained a saturable plasma concentration of sildenafil for the PDE-5 receptor $(>>3.5 \mathrm{nM})$ at all time points (49). As concentrations of sildenafil that would saturate the PDE-5 enzyme were attained, any minor impact of pregabalin on the PK of sildenafil would be negligible in this study design.

An additional objective was to determine a PK sampling strategy sufficient for representing actual rat PK profiles during future planned PD experiments. Capturing individual PK would not be straight-forward, as blood sampling during the PD experiment could lead to an introduction of PD measurement errors. For this reason, simulations provide an ideal platform for evaluating multiple limited sampling strategies for describing individual PK $(17,44,50)$. Furthermore, this type of preclinical trial simulation allows efficient examinations of multiple experimental scenarios before any animals are utilized, ensuring that experimental animals are utilized in the most effective manner possible.

A total of eight sampling scenarios were examined by combining PK experimental data with simulated rat pregabalin plasma concentrations based on the above developed pregabalin PK model. Sampling strategy success was then assessed by comparing the PE of the predicted clearance to the actual (combined experimental and simulated data set) population clearance. Sampling the same rats on another occasion was determined to be of no added value compared to taking no blood samples at all due to the large degree of intra-individual and inter-occasion variability identified in this PK model for the volume $(0.015(9.0 \%))$ and clearance $(0.098$ (27.1)\%). PK predictions using no blood samples from any of the rats produced no bias in the estimates of clearance, but it introduced a lack of precision in estimating individual PK. Optimally, taking eight blood samples spaced during and after the experiment produced the lowest bias and highest precision in the prediction errors.

Sampling in this optimal fashion described above served only as a benchmark, since collecting blood samples during the PD experiment would confound the PD experimental results. For this reason, additional sampling strategies involving a varying number of post-PD samples were also examined. Collecting one post-PD blood sample offered a small improvement in precision over no blood sampling, but it also introduced bias into the prediction errors. Increasing the number of post-PD samples reduced this bias and improved the precision; however, the degree of improvement in both bias and precision diminished with increasing blood samples. Increasing from three to eight post-PD samples slightly improved prediction error precision, but it was decided that these improvements in precision were not sufficient to warrant the collection of five additional samples due to the resulting blood volume alterations, animal stress, and research costs. Thus, collecting three post-PD blood samples was deemed sufficient for removing bias in the prediction error and drastically improving the precision.

Static allodynia PD simulations supported this conclusion by demonstrating that the three-sample schedule provided increased precision in estimating EC50s compared to taking no blood samples, and was only marginally worse than the unrealistic best-case scenario of eight samples taken during and after the PD experiment. This increased precision in identifying individual EC50s will allow a greater ability to identify changes in potency when examining the interaction between these two compounds. Furthermore, these types of simulations may also serve as an example for other preclinical studies to help refine experimental design.

Overall, this study developed a two-compartment model capable of accurately describing pregabalin PK in the presence or absence of sildenafil. These results also served to inform the sampling schedule design for future quantitative PK-PD study examining the interaction of these two drugs. Finally, this PK study also served as an initial step towards identifying and quantifying potential PK and PD interactions between pregabalin and sildenafil.

\section{ACKNOWLEDGEMENTS}

The authors thank Kenneth Watson and Pawel Dzygiel within the department of PDM, Pfizer, Sandwich, U.K. for practical contributions in the characterization of the bioanalytical assay and associated sample analysis.

Open Access This article is distributed under the terms of the Creative Commons Attribution Noncommercial License which permits any noncommercial use, distribution, and reproduction in any medium, provided the original author(s) and source are credited.

\section{REFERENCES}

1. Jensen MP, Chodroff MJ, Dworkin RH. The impact of neuropathic pain on health-related quality of life: review and implications. Neurology. 2007;68(15):1178-82.

2. Chong MS, Brandner B. Neuropathic agents and pain. New strategies. Biomed Pharmacother. 2006;60(7):318-22.

3. Francis DA, Christopher AT, Beasley BD. Conservative treatment of peripheral neuropathy and neuropathic pain. Clin Podiatr Med Surg. 2006;23(3):509-30.

4. Gilron I, et al. Neuropathic pain: a practical guide for the clinician. CMAJ. 2006;175(3):265-75.

5. Jackson KC II. Pharmacotherapy for neuropathic pain. Pain Pract. 2006;6(1):27-33.

6. Nurmikko TJ, Haanpaa M. Treatment of postherpetic neuralgia. Curr Pain Headache Rep. 2005;9(3):161-7.

7. Stillman M. Clinical approach to patients with neuropathic pain. C Cleve Clin J Med. 2006;73(8):726-8. 729-30, 733-6 passim.

8. Harden N, Cohen M. Unmet needs in the management of neuropathic pain. J Pain Symptom Manage. 2003;25(5):S12-7. Supp 1.

9. Backonja MM, Irving G, Argoff C. Rational multidrug therapy in the treatment of neuropathic pain. Curr Pain Headache Rep. 2006;10(1):34-8.

10. Gilron I, Max MB. Combination pharmacotherapy for neuropathic pain: current evidence and future directions. Expert Rev Neurother. 2005;5(6):823-30.

11. Jonker DM, et al. Towards a mechanism-based analysis of pharmacodynamic drug-drug interactions in vivo. Pharmacol Ther. 2005;106(1):1-18.

12. Chien JY, et al. Pharmacokinetics/Pharmacodynamics and the stages of drug development: role of modeling and simulation. AAPS Journal. 2005;7(3):E544-59. 
13. Miller R, et al. How modeling and simulation have enhanced decision making in new drug development. J Pharmacokinet Pharmacodyn. 2005;32(2):185-97.

14. Jonker DM, et al. Synergistic combinations of anticonvulsant agents: what is the evidence from animal experiments? Epilepsia. 2007;48(3):412-34.

15. Aarons L, et al. Practical experience and issues in designing and performing population pharmacokinetic/pharmacodynamic studies. Eur J Clin Pharmacol. 1996;49(4):251-4.

16. Geldof $\mathrm{M}$, et al. Population pharmacokinetic model of fluvoxamine in rats: utility for application in animal behavioral studies. Eur $\mathrm{J}$ Pharm Sci. 2007;30(1):45-55.

17. Ette EI, et al. Experimental design and efficient parameter estimation in preclinical pharmacokinetic studies. Pharm Res. 1995;12(5):729-37.

18. Ette EI, et al. Analysis of animal pharmacokinetic data: performance of the one point per animal design. J Pharmacokinet Biopharm. 1995;23(6):551-66.

19. Anonymous. Pregabalin (Pfizer). Curr Opin Investig Drugs. 2004;5(1):82-9.

20. Zareba G, Zareba G. Pregabalin: a new agent for the treatment of neuropathic pain. Drugs Today (Barc). 2005;41(8):509-16.

21. Guay DR, Guay DRP. Pregabalin in neuropathic pain: a more "pharmaceutically elegant" gabapentin? Am J Geriatr Pharmacother. 2005;3(4):274-87.

22. Lauria-Horner BA, et al. Pregabalin: a new anxiolytic. Expert Opin Investig Drugs. 2003;12(4):663-72.

23. Hitiris $\mathrm{N}$, et al. Modern antiepileptic drugs: guidelines and beyond. Curr Opin Neurol. 2006;19(2):175-80.

24. Hamandi K, et al. Pregabalin: a new antiepileptic drug for refractory epilepsy. Seizure. 2006;15(2):73-8.

25. Briganti $\mathrm{A}$, et al. Clinical update on phosphodiesterase type-5 inhibitors for erectile dysfunction. World J Urol. 2005;23(6):374-84.

26. Antoniu SA, Antoniu SA. Sildenafil citrate for the treatment of pulmonary arterial hypertension. Expert Opin Pharmacother. 2006;7(6):825-8.

27. Jackson G, et al. Past, present, and future: a 7-year update of Viagra (sildenafil citrate). Int J Clin Pract. 2005;59(6):680-91.

28. Bender G, Gosset J, DeJongh J, Bies R, Danhof M. Simulations to guide mechanism based PK-PD moeling of a synergistic interaction in an animal model of neuropathic pain. Leiden Amsterdam Center for Drug Research Quadrenniel meeting on In-Vivo and In-Vitro Measurement and Modeling of Pharmcodynamics. Noordwijkerhout, Netherlands, April 26-29, 2006.

29. Meno-Tetang GM, et al. On the prediction of the human response: a recycled mechanistic pharmacokinetic/pharmacodynamic approach. Basic Clin Pharmacol Toxicol. 2005;96(3):182-92.

30. Gieschke R, Reigner BG, Steimer JL. Exploring clinical study design by computer simulation based on pharmacokinetic/ pharmacodynamic modelling. Int $\mathrm{J}$ Clin Pharmacol Ther. 1997;35(10):469-74.

31. Ette EI, Williams PJ. Population pharmacokinetics I: background, concepts, and models. Ann Pharmacother. 2004;38(10):1702-6.

32. Gieschke R, Steimer JL. Pharmacometrics: modelling and simulation tools to improve decision making in clinical drug development. Eur J Drug Metab Pharmacokinet. 2000;25(1):49-58.
33. Sheiner LB. The population approach to pharmacokinetic data analysis: rationale and standard data analysis methods. Drug Metab Rev. 1984;15(1-2):153-71.

34. Sheiner LB, Steimer JL. Pharmacokinetic/pharmacodynamic modeling in drug development. Annu Rev Pharmacol Toxicol. 2000;40:67-95.

35. Bennett GJ, et al. A peripheral mononeuropathy in rat that produces disorders of pain sensation like those seen in man. Pain. 1988;33(1):87-107.

36. D'Argenio D.Z., S.A., ADAPT II User's Guide: Pharmacokinetic/Pharmacodynamic Systems Analysis Software. 1997: Biomedical Simulations Resource, Los Angeles.

37. Sheiner LB, Rosenberg B, Marathe VV. Estimation of population characteristics of pharmacokinetic parameters from routine clinical data. J Pharmacokinet Biopharm. 1977;5(5):445-79.

38. Beal B, Sheiner L, NONMEM user's guide, Part I. San FranCIIsco: University of California at San FranCIIsco. 1992.

39. Wahlby U, Jonsson EN, Karlsson MO. Comparison of stepwise covariate model building strategies in population pharmacokineticpharmacodynamic analysis. AAPS Pharmsci. 2002;4(4):E27.

40. Beal SL. Commentary on significance levels for covariate effects in NONMEM. J Pharmacokinet Pharmacodyn. 2002;29(4):403-10. discussion 411-2.

41. Wahlby U, Jonsson EN, Karlsson MO. Assessment of actual significance levels for covariate effects in NONMEM. J Pharmacokinet Pharmacodyn. 2001;28(3):231-52.

42. Wade JR, Beal SL, Sambol NC. Interaction between structural, statistical, and covariate models in population pharmacokinetic analysis. J Pharmacokinet Biopharm. 1994;22(2):165-77.

43. Mandema JW, Verotta D, Sheiner LB. Building population pharmacokinetic-pharmacodynamic models. I. Models for covariate effects. J Pharmacokinet Biopharm. 1992;20(5):511-28.

44. Ette EI, et al. Model appropriateness and population pharmacokinetic modeling. J Clin Pharmacol. 2003;43(6):610-23.

45. Parke J, Holford NH, Charles BG. A procedure for generating bootstrap samples for the validation of nonlinear mixed-effects population models. Comput Methods Programs Biomed. 1999;59 (1):19-29.

46. Ette EI. Stability and performance of a population pharmacokinetic model. J Clin Pharmacol. 1997;37(6):486-95.

47. Yano Y, Beal SL, Sheiner LB. Evaluating pharmacokinetic/ pharmacodynamic models using the posterior predictive check. J Pharmacokinet Pharmacodyn. 2001;28(2):171-92.

48. Cooper JDH, Muirhead DC, Taylore JE, Baker PR. Development of an assay for the simultaneous determination of sildenafil (Viagra) and its metabolite (UK-103,320) using automated sequential trace enrichment of dialysates and high performance liquid chromatography. J Chrom B. 1997;701:8795.

49. Pauver O, Lugnier C, Keravis T, Marthan R, Rousseau E, Savineau JP. Effect of sildenafil on cyclic nucleotide phosphodiesterase activity, vascular tone and calcium signaling in rat pulmonary artery. Br J Pharamcol. 2003;139(3):513-22.

50. Roy A, Ette EI. A pragmatic approach to the design of population pharmacokinetic studies. AAPS Journal. 2005;7(2): E408-20. 